\title{
Addressing Health Crisis among Pregnant Women with Disabilities: From the Zimbabwean and Zambian Point of View
}

\section{Chigunwe $\mathrm{G}^{1 *}$ and Mphande $\mathrm{F}^{2}$}

${ }^{1}$ Department of Disability Studies and Special Needs Education, Zimbabwe Open University, Zimbabwe

${ }^{2}$ Department of Education and Sociology, University of Africa, Zambia

*Corresponding author: Gilliet Chigunwe, Department of Disability Studies and Special Needs Education, Zimbabwe Open University, Zimbabwe, Tel: +26377331338; Email: gchigunwe@gmail.com; chigunweg@zou. ac.zW

\section{Abstract}

Projects that address changing values in our communities and that have impact on needs technology as well as addressing the health for all should be considered in all African countries. COVID19 is a point of argument. During this study, African leaders were running around for intervention measures and protection of their people from Corona virus. One wonders if the state of urgency would have been the same if COVID19 affected only those who cannot communicate such as the poor and those with disabilities. The latter minority groups have always been dying of preventable diseases such as Malaria and malnutrition whilst most women with disabilities experience maternal challenges in most African countries. Zimbabwean government indulged in the construction of alternative sanitary facilities in schools, clinics and hospitals.

This move alleviated the sanitary and hygiene challenges faced by Zimbabweans. Studies revealed that persons with physical disabilities accessed most of these public institution toilets by leaving their wheelchairs outside then crawl on their hands and feet despite the dirty toilet floors. Most public toilet corridors were too narrow for wheelchairs to manoeuvre and there were no rails for support. In a study to examine the coping mechanisms and adaptation of women with physical disabilities in such an environment, qualitative methodology was used. Ethnography was used to gather data in Bindura district of Zimbabwe and Lusaka district of Zambia. Cross-sectional research was employed and interviews and questionnaire were data collection instruments.

It was found that difficulty in accessing health institutions buildings and sanitary facilities made women with disabilities indulge in alternative, unhealthy practices which included home pregnancy delivery as well as failure to take their babies for baby health check clinic. While the UN Convention on the Rights of Persons with Disabilities emphasis on inclusive health, rehabilitating all institutions cannot be achieved now in Zimbabwe and Zambia due to economic crisis. However, SDGs can still be met by adopting policies that ensure healthy lives and wellbeing for all. Pregnant Women with Disabilities Community Care Model (PWwDCCM) can be adopted as an integrated home care model. This would be a holistic approach to managing pregnant women with disabilities as well as participation of health personnel and community members. Thus the two countries can contribute significantly in reducing the global maternal mortality ratio as well as preventing deaths of new born babies.

Keywords: Pregnant women with disabilities; Access; Healthcare Facilities; Maternal Deaths; Home Care Model 


\section{Introduction}

Addressing health crisis in Africa calls for practices which provide flexibility to health care organisations and adaptation of sustainable innovations. Thus projects that address changing values in our towns and cities and that have impact on needs technology as well as addressing the health for all should be considered. Studies carried out in Zimbabwe revealed that persons with physical disabilities have had problems in accessing sanitary facilities due to infrastructure designs which are exclusive of such persons, especially pit latrines and water pumps such as boreholes [1]. Dams in Zimbabwe were failing to supply cities and towns because of continuous little rains. The situation negatively affected the functions of water system toilets. To alleviate the sanitary and hygiene challenges, the Government of Zimbabwe in conjunction with non-governmental organizations constructed boreholes and ventilated latrine toilets in Zimbabwe from 2007 to date. Whilst these developments were a noble cause for ameliorating sanitary challenges Zimbabweans were facing, they left out persons with physical disabilities. Most public sanitary facilities are inaccessible or difficult to access by persons with physical disabilities.

Studies revealed that persons with physical disabilities accessed most of these public institution toilets by leaving their wheelchairs outside then crawl on their hands and feet despite the dirty toilet floors. Corridors were too narrow for wheelchairs to manoeuvre and there were no rails for support. Some toilets, for example, the pit latrines had no seaters but holes and these are the most common type of toilets which were made to substitute flash toilets in most public institutions of Zimbabwe (ibid). These public institutions included schools, clinics and hospitals.

Our observation during this study was that, access to health facilities is a challenge not only to Zimbabweans with disabilities but to Zambians with disabilities too. Public health infrastructures in both countries are difficult to access by men and women in wheelchairs. Inaccessibility challenges were noted to have much bearing on women because of their menstrual cycles that demands access to sanitary facilities as well as pregnancies that demands access to healthcare facilities. Despite the persistence of social determinants, resource barriers to service access and social barriers to uptake of services, the gains in maternal and neonatal and child survival, morbidity and mortality remain high in Zimbabwe and Zambia [2]. Because women's health is determined by the environment and social and economic circumstances in which they live, addressing women's health is a key element to reducing maternal health and child mortality in both countries. Of interest to note is that, more women with physical disabilities in Zimbabwe and Zambia are increasingly choosing to become pregnant.

Whilst this was so, it appears no studies have been carried out to assess coping mechanisms of pregnant women with physical disabilities where healthcare facilities are difficult to access in Zimbabwe and Zambia. It is against this background that this study intended to assess coping mechanisms of pregnant women with physical disabilities where healthcare facilities are difficult to access.

\section{Review of Related Literature}

Women's health is the foundation for social and economic development in the African region [3]. It is recognisable as a human rights issue and should be promoted and defended as such. According to World Health Organisation [2] women in Africa represent slightly over $50 \%$ of the continent's human resources and so women's health has huge implications for the Region's development. Focusing in particular on the unacceptably high level of maternal mortality in subSaharan Africa, the latter calls for a fundamental rethinking of approaches to improving women's health informed by an understanding of the sociocultural determinants that are so important in shaping it. A core contention of WHO $[3,4]$ is that a range of adverse socioeconomic pressures including inadequate health care prevents African women from realising their full potential.

It is evident that the interventions to improving women's health, focusing solely on "public health" issues miss the fundamental interconnectedness of health with other factors in society; recognising this interconnectedness is the starting point for emphasizing the multispectral approach required in the African region. To shed light on the often complex relationships between women's health and their socioeconomic status, it's time researches on women's health took an inclusive and multidisciplinary approach to evidence gathering as well as analysis. A gap in literature is noted in the adoption of a life course approach to women's health to reveal the specific challenges faced by heterogeneity of African women. A number of researches reviewed fill in the gap of women's health and accessibility to healthcare facilities but leave out women with disabilities.

Of course a number of researches have been carried out in the African community pertaining to women with disabilities as is the case of Rugoho, et al. [5-7] to mention some. The latter scholars looked at accessibility of women with disabilities to sexual health in Zimbabwe as well as accessibility of women with disabilities to maternal healthcare services in Ghana, respectively. However, research seems grey in areas of coping mechanism for women with disabilities in communities where healthcare facilities are inaccessible or difficult to access. 
Through review of literature on public health, it appears there are issues that have often been neglected when drawing up and implementing public health policies in most African countries, especially policies directed at improving women's health. Underlining the many development opportunities in Africa, we deduce that, with appropriate intervention strategies and sustained investment, a profound impact on all women's health and their socioeconomic status in Africa and this includes those with disabilities.

One of the biggest problems faced by the Region's health systems is insufficient numbers of qualified and motivated health workers. According to WHO and World Bank [4] data, 36 of the 57 countries worldwide facing a critical shortage of health workers are located in Africa. Poor working conditions and inadequate pay are two of the main reasons for this, but staff recruitment and retention are also compromised by political instability, ongoing financial crises and the HIV/ AIDS epidemic (ibid).

According to WHO [8] one woman dies per minute in childbirth around the globe. Almost half of these deaths occur in Sub-Saharan Africa. Despite the progress made in many countries in increasing the availability of maternal healthcare, the majority of women across Africa remain without full access to this care thus causing high maternal mortality in Africa $[2,9,10]$. World Health Organization [9] defines the maternal mortality ratio (MMR) as the annual number of female deaths from any cause related to or aggravated by pregnancy or its management (excluding accidental or incidental causes) during pregnancy and childbirth or within 42 days of termination of pregnancy, irrespective of the duration and site of the pregnancy, per 100,000 live births, for a specified year. In Zimbabwe, Zimstat calculates the Maternal Mortality Rate by dividing the number of maternal deaths by the number of women aged 15-49 years. Countries face a variety of obstacles to improved maternal health: insufficient data prevents ministries from implementing programmes most effectively, while cost and other access issues prevent women from using the available resources [11].

According to UNDP [12] in the context of reproductive health, women with disabilities have largely been ignored in reproductive health research and programming. Studies by Morrison, et al. $[6,13,14]$ on disability in sexual and reproductive health policies and research in Ghana concluded that persons with people with disabilities have received little attention. Part of the reason for this neglect is that they are often thought not to be sexually active, and less likely to marry or to have children than women without disabilities.

The perception that women with disabilities are not sexually active has often resulted in provision of limited access to health services for them. A number of studies by Alwan, et al. [15-18] have suggested negative social attitudes and cultural assumptions such as the equation to being normal and not disabled, physically inaccessible health facilities, insensitivity of healthcare providers, limited knowledge by healthcare providers about disability, and limited information tailored to their health needs as factors hampering access to skilled healthcare services by women with disabilities.

Whilst persons with disabilities continue to be neglected in most African countries [10,19] issues of disabilities have been included in various targets and as a cross-cutting issue in the 2030 Agenda for Sustainable Development. Efforts need to be stepped up to ensure that the goals and targets will be achieved for persons with disabilities too, in line with the United Nations Conventions on the Right of Persons with Disabilities [20]. Of interest to note is that, SDG numbers 1 and2 emphasises on alleviating poverty and hunger whilst health and well-being is stipulated in SDG 3, sexual and reproductive health and reproductive rights targets 3.7 and 5.6, education, SDG; gender equality and empowerment of women and girls with disabilities; SDG 5, whilst SDG number 6 articulate on the availability of water and sanitation. Standard Development Goal number 3 shows that health is a fundamental human right, encompassing access to quality health services and other underlying social determinants of health [11]. The greatest gains in health worldwide have been achieved through improvements outside health service provision, such as education, working conditions, accommodation and housing, food safety, water supplies, waste management and the physical environment, including improved transport and access [3].

Gender inequalities are important causes of inequality in health and health care. It has been argued that gender inequalities are one of the most determinants of health. This is because gender roles, which are socially and culturally determined, influence the different behaviour, roles, responsibilities and expectations of men and women [12]. These expectations influence whose needs and priorities are addressed even in the health system. This can mean that the needs of women and girls are not addressed. Whilst this so, women and girls with disabilities are worse off in terms of marginalisation. This marginalisation extends to access to sexual health and reproduction.

\section{Research Methodology}

The study was based on qualitative methodology and ethnography method was used to gather data. Ethnography mainly focuses on the ontology and behaviours of the researched community. Interviews and observation were used to gather data among women with disabilities in Bindura District of Zimbabwe 
and Lusaka District of Zambia. Through 'snow bowling' technique, women with physical disabilities were conveniently selected for face to face interviews as well as through WhatsApp calls. Data gathered during interviews saturated on the $6^{\text {th }}$ woman in Zimbabwe and $5^{\text {th }}$ woman in Zambia. Attride-Stirling's thematic network framework was used to analyse the data.

The following three principles of ethics guided this research; consent, confidentiality and anonymity. Consent was sought from Traditional Birth Assistant (TBA) for photo shooting so that the pictures get published. More so, consent was also sought from participants to record their interviews. More so, informants were assured of confidentiality before responding to interview questions. They were assured of anonymity by the researcher. Scientifically acceptable research protocols that were worth the informants' time and had a reasonable chance of yielding meaningful findings were developed. The rights of the informants (stakeholders and persons with disabilities) were observed in the research process. Hence findings were reported fairly and accurately and it was the researcher's intention to cooperate with other academics or researchers and share findings of her research. The research was grounded in the international policies on inclusivity such as the United Nations Declaration Charter on the right of persons with disabilities and the Standard Development Goals (SDGs).

\section{Findings}

Structured interviews were administered with conveniently selected women with disabilities in Bindura, Zimbabwe and Lusaka, Zambia among women with disabilities. The idea was to source deeper understanding of the coping mechanisms of pregnant women with physical disabilities where healthcare facilities are difficult to access in Zimbabwe and Zambia. It was about harvesting their deeper feelings and emotions as they discussed. Data saturation was reached at the $6^{\text {th }}$ interviewee in Zimbabwe and $5^{\text {th }}$ interviewee in Zambia. Below is Table 1 showing the profile of participants who were interviewed.

\begin{tabular}{|c|c|c|c|c|c|}
\hline Name & Occupation & Work Experience & Marital Status & Nature of Disability & Age \\
\hline Tendo & None & None & single & congenital & 29 \\
\hline Farai & None & None & single & congenital & 33 \\
\hline Mary & None & None & single & congenital & 35 \\
\hline Kudzi & None & None & single & congenital & 30 \\
\hline Mbata & Self employed & 5 years & married & Acquired & 28 \\
\hline Zai & None & None & single & congenital & 35 \\
\hline Bupe & None & None & single & Acquired & 33 \\
\hline Kanunka & None & None & single & congenital & 30 \\
\hline Rute & None & None & single & congenita & 29 \\
\hline Ibondwe & None & None & single & congenital & 44 \\
\hline Kabwita & None & None & Married & Acquired & 48 \\
\hline
\end{tabular}

Source: Researchers, 2020.

Table 1: Presents Profile of Women with Disabilities Participants.

Interviews were set to examine the coping mechanisms and adaptation of pregnant women with physical disabilities in situations where they had difficulties in accessing sanitary facilities at clinics and hospitals. These interviews were captured verbatim in Shona and Bemba. (Shona is one of the main native languages in Zimbabwe whilst Bemba is Zambian main languages). The responses were transcribed in English as presented. The following responses were captured;

Tendo: From the time I fell in the dirty floors of the hospital toilet, I never set foot there. I have given birth to my two children home. Nyamukuta (traditional midwifery) in our neighbourhood assisted me to deliver.

Farai: It is not easy to access toilets in clinics and hospitals thus we prefer to deliver in homes where we easily access toilets.

Mary: Yes there is good hygiene at health institutions but when accessibility of those places is not easy, better to find alternatives that accommodate us. Homes are better. At least we are given good care, for example, we are carried to toilet when need be by our family members an act that is not common among nurses in hospitals and clinics.

Kudzi: In my home area, there is Blair toilets at our local clinic, my wheelchair can't enter. More so entering healthcare buildings in wheelchair is very difficult as there are steps at entrance. Hence better to stay home than bothering myself.

Mbata: I gave birth to a beautiful baby girl at home. She is now doing grade 5 , its unfortunate she is not around, you 
could have seen her. She is a very healthy girl but I never took her for baby clinic as what able bodied people do to their babies. The reason why I did not take her for baby clinic was that, it is not easy for me to travel to clinics in wheelchair, with these bad roads. Minibuses demand that I pay fares for two because of my wheelchair which they say occupies more space. Suppose I reach the health care institutions, buildings are not easy to access in wheelchair and worse, toilets are a challenge to enter.

Zai: I gave birth in hospital but I attended antenatal clinic once. As you know, we people in wheelchairs experience incontinency hence we visit toilets frequently. Now, problems in accessing toilets at our local hospital made me decide to nurse my pregnancy home. I delivered at hospital but could not take baby for baby clinic. Family members took baby for me to be checked on the first ten days and after six weeks. Thereafter, they stopped. I could not take her myself because I am not comfortable to be away from home for many hours. If the call of nature demands that I visit the toilet out there, I experience nightmare since most public toilets are not easy to access, worse the clinic and hospital ones.

Bupe: Aaaa Mother, clinic and hospital here are very difficult to access. This has made most of us in this condition fail to go for treatments when we are sick. Better to send someone to buy you drags over counter. If the needed drags demand doctor prescription, then that is it. I just pray for God's intervention.

Kanunka: Hospitals are not meant for us mother, they are for able bodied. If you think am lying visit any hospital or clinic here and see if they had us in mind when they did the constructions. Able bodied people are very selfish. That's the truth mother, am not insulting you.

Rute: Hospitals and clinics are for the able-bodied, we force ourselves there. As for me, I have since stopped to go where I am not wanted. I have resorted to prayers, I believe in God's intervention over my health thus I use natural herbs.

Ibondwe: Mmmm going to hospital is a mammoth task with these bad roads of ours. Public transport shun us from boarding, most say they have no space for wheelchairs. I have personally resorted to receiving healthcare home. I bore my child \& raised him here. We are all health; God is taking care of us in these unfriendly environments.

Kabwita: Most of us mother resort to home treatment. I wish our health personnel would consider building a special hospital for us, these are healthcare institutions do not accommodate us. Most staff doesn't understand our situation. They project to us the fact that we are asexual and not worthy to become mothers.

Kabichi: We don't have a place in this world, they hate us so no point in telling you on how we cope. Who cares? I bore my child home; unfortunately I lost her after two days. Life goes on as I await God to take his spirit.

The following themes were generated from the interviews;

- Failure for women with physical disabilities and their babies to access general treatment in healthcare institutions have led them to find alternatives, viz;

$>$ giving birth in homes

$>$ Seeking Traditional Birth Assistance (TBA) to help in baby delivering process.

$>$ Absconding anti-natal clinics.

$>$ Failure to take new born babies for baby clinics and immunization process.

The following data was also generated from interviews;

- Pregnant women with disabilities encountered negative experiences from some of the Healthcare providers who doubted their ability to become pregnant and mothers.

- Prenatal classes were found uninformative and not helpful by pregnant women with disabilities who attempted to attend prenatal classes because the class instructors were unknowledgeable about their disabilities as well as on the possible effects of their pregnancy, labour and delivery issues.

- Most women with physical disabilities experienced difficulties to recover during postpartum because of inaccessibility of the physical healthcare environment which included sanitary facilities.

\section{Home Maternal Delivery}

In an interview, one of the TBAs confidently pointed out that she delivers a minimum of 5 babies per month and she encounters an average of 2 complication and death per 6 months.

Below are some pictures of one of the homes where vulnerable women and those with disabilities are assisted in giving birth to babies in Zimbabwe.

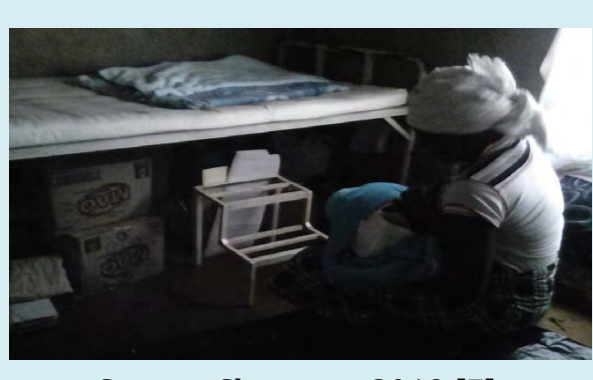

Source: Chigunwe 2019 [7].

Figure 1: A woman breastfeeding a newly born baby delivered in the home. 


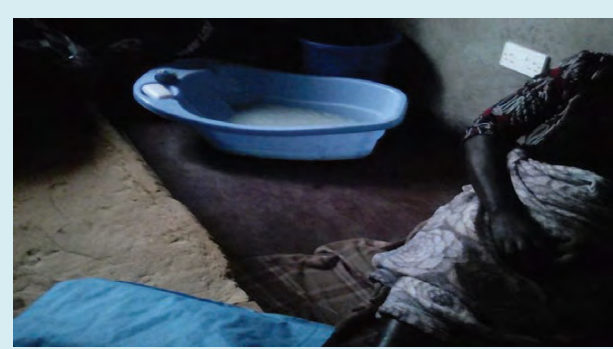

Source: Chigunwe 2019 [7].

Figure 2: Seated in home labour room is the woman who assists in delivering of babies.

\section{Discussion}

Maternal health is a key part of the right to health, for both mother and unborn child. Safe Motherhood means creating circumstances that ensure:

- A pregnant woman is able to receive care for prevention, detection and treatment of pregnancy complications;

- A woman in labour has access to skilled birth attendants; and

- If they need it, mother and newborn have access to emergency obstetric care and care after birth to prevent death or disability from any other complication. .

While provisions of effective and accessible maternity services are vital to prevent women from dying during childbirth, pregnant women with disabilities remain vulnerable to maternal immortality as access to healthcare facilities remain a challenge in Zimbabwe and Zambia.

Of course both countries have made some strides in improving maternal health as seen in the 2007 Zambia Demographic Health Survey [11]. The maternal mortality ratio (MMR) for Zambia decreased from 729/100,000 to $591 / 100,000$ live births by 2014. As for Zimbabwe, maternal mortality ratio (MMR) has declined from 960 deaths per 100,000 live births in 2010 to 614 deaths per 100,000 live births in 2014 [20-22]. The two countries have made commendable progress though the ratio still remains unacceptably high. Zimbabwe's SDG 3 target is to reduce MMR to 71 deaths per 100,000 live births by 2015 whilst Zambia intended to reduce to $120 / 100000$ by 2015 (ibid). This suggest that there is need for the Ministry of Health in both countries to monitor progress and to put in place measurement tools that assess as to what level maternal deaths have been reduced by 2020 . The media should also play a critical role in promoting social accountability on maternal health so that communities participate in reducing neonatal mortality rate. The World Health Organisation [19] defines the neonatal mortality rate as deaths among live births during the first 28 completed days of life. These may be subdivided into early neonatal deaths, occurring during the first 7 days of life, and late neonatal deaths [23].

Further, a decline in maternal mortality rates depends on scaling up as well as making universally accessible wellproven maternal and new born interventions. This includes increasing access to emergency obstetric and new born care, skilled attendants during pregnancy, childbirth, and postnatal period and access to family planning. The study noted that this access to and uptake of services for women living in poverty is yet to be achieved. It was found that pregnant women with disabilities had challenges in accessing healthcare facilities thus resorted to delivering their babies in homes with the assistance of Traditional Midwifery or Traditional Birth Assistants. Thus to cover this gap, processes and practices that provide flexibility to healthcare access and adaptation of sustainable innovations should be put in place by Zimbabwe and Zambia as well as other countries in Africa who have similar challenges. Lack of access of a certain group of people to healthcare, as is the case of pregnant women with physical disabilities, is a violation of human rights and havoc to the nation. Such blunder may thwart countries to achieve the SDGs as stipulated by article number 3 . More so, the Africa we want goals may not be achieved.

The argument is that, failure of the health systems in the majority of African countries to provide accessible care of adequate quality is one of the main drivers of the adverse trends in women's health indicators. This situation stems from underinvestment in women's health and also from other factors such as inadequate empowerment of women and poor health systems design. Since 2003, average health spending as a percentage of total spending by African countries has hovered around $10 \%$, that is, two thirds of the level to which African leaders committed themselves in Abuja in 2001. It is worth noting that over ten years after Abuja only Botswana, Burkina Faso, Democratic Republic of Congo, Liberia, Rwanda, Tanzania and Zambia are delivering on their pledges [8] while 13 African countries actually allocate less of their total government budgets to health now than they did prior to 2001. This implies that, Zimbabwe is under delivering on its pledges in terms of healthcare delivery. Hence one can deduce that, even with adequate funding, health system in some countries like Zimbabwe will struggle to meet the needs of women unless fundamental changes are made in health systems design. The majority of modern health care services provided in Zimbabwe and Zambia were observed to be clinic-based, physician-oriented and urban centred, leaving the predominantly rural population woefully underserved. It is therefore crucial that policy makers in the two countries understudy rethink health systems design, placing greater emphasis on primary health care (PHC). The organization of maternal health care delivery in particular needs to be reconsidered and reorganized with a view to improving access to basic and comprehensive emergency 
obstetric care by all women, able bodied and disabled. Whilst it was observed that both countries under study had maternity hospitals specifically designed to deliver babies, these healthcare services were designed to meet the needs of women without disabilities. The infrastructures lacked flexibility and responsiveness to meet the unique maternity care needs of women with disabilities.

If Zimbabwe and Zambia and other African countries so wish to fulfill their obligation to the right to health for all as well as to attain the health related SDGs by 2030, resources must be proactively allocated to support the most vulnerable and underserved segment of the population including women with disabilities. Investing in maternal health is a wise health and economic policy decision. Women are the sole incomeearners in nearly one third of all households globally. There are spill-over macro-economic benefits from the women whose lives are improved by maternal health interventions. Maternal care intervention for women with disabilities maternal care interventions can be both effective in reducing maternal death and cost-effective, especially for high-risk groups. Some of these interventions are cost-saving, yielding returns of investments especially on the government social welfare services.

Thus more investment should be done in healthcare delivery, system, eliminating preventable deaths, improving social safety networks for the poor and marginalized. COVID19 is a point of argument. A recent paper by Broadbent, et al. estimated a $0.32 \%$ fatality rate in its study population of people aged 60 under and 3\% of the population over the age of 65 worlds over. The median age in Africa was noted to be 18 whilst that of Europe was noted to be 42 . Thus for argument sake, during the time of this study, COVID19 threat seemed less serious in Africa than elsewhere but African leaders are running around for intervention measures and protection. The Chief of Health at UNICEF (ibid) had it that, countries are running around to find measures to control the COVID19 virus because the virus is affecting the people with power, the people who can communicate, rather than the poor who have always been dying of preventable diseases such as Malaria and malnutrition. The lockdown situations in most African countries are going through should mandate stakeholders to redirect priorities in as far as healthcare is concerned. More attention should be accorded to vulnerable persons with disabilities such that as renovation and equipping of hospitals take place in Zimbabwe and Zambia, inclusive policies of access to healthcare should be considered. Inclusive models should be put into priority, especially those models that reduce deaths among the most vulnerable as well as healthcare models that are specifically designed for pregnant women with disabilities.

Pregnant Women with Disability Community Care Model
(PWwDCCM) can be adopted as an integrated home care model by Zimbabwe and Zambia. This would be a holistic approach to managing pregnant women with disabilities as well as participation of health personnel and community members. The distinctive feature of this home care model is that, it would offer more personalised and need sensitive care and it would assign pregnant women with disabilities to primary care physicians thus ensuring patients know who is responsible for their health and the providers would know the patients they are responsible for. A coordinator would be in place to assess the special needs of patients, develop an individual care plan and to coordinate the provision of appropriate service. This health professional coordinator will be networking with Traditional Midwifery (TM) or Traditional Baby Assistance (TBAs) who are in communities since these are the women assisting most persons with disabilities as are the findings of this study.

What we saw as a missing element during our study is the issue of equipping TBAs with delivery equipments they require. Zimbabwe differs from Zambia in that, it once designed a programme to train TBAs through the Ministry of Health throughout the country. As for Zambia, TBAs were trained in the form of a pilot project in Lufwanyama and Chongwe in Lusaka district [16]. However, despite training efforts in both countries, a gap was noted in that, the programme did not prepare TBAs to assist pregnant women with disabilities and these women with disabilities are found going to seek assistance of TBAs during delivery stages. Ironically, the moment a woman in labour comes for assistance; African religious beliefs as well as cultural demands compel the TBA to render the needed assistance. Such scenario exposes women with disabilities and their babies to high risk of maternal deaths in Zimbabwe and Zambia. Infact, this study found that, for many women living in Zimbabwe and Zambia, antenatal care as well as institutional deliveries with skilled health workers remains a distant reality. Inclination towards home births supervised by TBAs is associated with cultural norms and religious beliefs as well as costs and accessibility of services.

\section{Conclusion of the Study}

The study revealed that most women with disabilities who faced challenges in accessing healthcare facilities sought traditional healers and traditional midwifery as alternatives. It was noted that the majority of women with disabilities give birth in homes with the assistance of Traditional Midwifery (TM) or Traditional Birth Assistance (TBA). Most women complained of negative attitude projected towards them by health professionals as they viewed them incapable of becoming mothers. Inaccessible infrastructures of healthcare facilities attributed to their seeking of alternatives too. Moreso, financial challenges to pay for healthcare has 
seen most women with disabilities opt for assistance for baby delivery from traditional midwifery in their locality who usually assist free of charge. The majority of women with disabilities in Zimbabwe and Zambia delivered in homes with the support of experienced women or TBAs. The study concluded that, despite the increasing number of States ratifying the United Nations Convention on the Right of Persons with Disabilities (UNCRPD) and the steps these countries have taken to implement article 25 of the UNCRPD, persons with disabilities continue to experience unmet health needs and barriers to accessing health services in comparison to the general population. Persons with disabilities continue to experience poorer health and poorer mental health in Zimbabwe and Zambia. More so, they continue to face barriers healthcare services in both countries under study. All these constitute a genuine obstacle to the implementation of Goal number 3 of the Standard Development Goals. Thus an urgent need for the Zimbabwe and Zambia to develop an inclusive cost effective health care model that encompasses the informal community structures that actively participate in maternal delivery process.

\section{Addressing Challenges Experienced by Pregnant Women with Disabilities in Accessing Healthcare Facilities}

Thus basing on the findings, this study points out for the necessity of developing a Pregnant Women with Disabilities Community Care Model (PWwDCCM). This model can go a long way in reducing unmonitored baby delivery by the Ministry of Health as well as reduction of maternal mortality among women with disabilities. Ministry of Health for Zimbabwe and Zambia should develop an inclusive training where health personnel are taught basics disability issues so as to improve service delivery for all. In other words, disability-inclusive education should be integrated into the curriculum and training for health professionals by the Ministry of Health of the countries in question. More so, strategies for holistic, people-centred care should be developed so as to improve the quality and continuity of care for all pregnant women. Hence, we propose establishment of a healthcare model that is inclusive of traditional birth assistance. It was noted that the majority of women with disabilities in both Zimbabwe and Zambia have developed coping mechanisms of substituting health professionals with TMs and TBAs. Of interest to note is that, in sub-Saharan African countries many child bearing women are still being attended by traditional birth attendants (TBAs) and relatives at deliveries. This implies that the practice of home delivery is not peculiar to Zimbabwe and Zambia but an African practice that has been there time immemorial. More so, literature shows that births without skilled personnel and without access to life-saving drugs are the commonest practice for millions of mothers in the poorest countries where mortality rates and morbidity of the mothers are the highest. African traditional healthcare is grounded in thousands of years of knowledge and has sustained life, on its own or in concert with Western medicine.

A traditional birth attendant is defined as a person who assists the mother during childbirth and initially acquired her skills by delivering babies herself or through apprenticeship to other TBAs [15]. According to Kruske, et al. [17] approximately half of all births in developing countries are attended by traditional birth attendants (TBAs) and as many as $95 \%$ ofwomen are attended by TBAs. It is against this background that we proposes that, rather than continuing to develop interventions grounded in a Western medicine world view of healthcare there is need to develop programs that are inclusive of healers who reflect the sociocultural beliefs of the community. There is also need to understand the local context and value traditional knowledge systems. Such calls are themselves not a new phenomenon; for example, WHO [21] called for the replacement of top-down, culturally inappropriate, bio medically oriented models with those of mutual accommodation. This is what Graham, et al. [20] calls the "partnership paradigm," that is, the mutual cooperation of biomedical and indigenous systems. However, it is at the interface between biomedical (Western) and indigenous systems that problems arise and also lie. The worldwide hegemony of Western biomedicine makes mutual accommodation an elusive goal.

The implication of the above discussion is that while an integral part of the health system, the ambivalence in terms of policy on the part of the Zimbabwe and Zambian government leave out TBAs in a predicament, they play a critical role in the baby delivery of women living in poverty especially women with disabilities. Extreme poverty is a factor in women with disabilities health. This is because most women with disabilities are denied access to education in most African communities. Social-cultural values among. Africans place more value on the education of able bodied children than those with disabilities. Thus lack of education has seen most persons with disabilities fail to compete with able bodied persons in the job market. Thus due to lack of empowerment in terms of education, most women with disabilities are among the population living below the poverty datum line in Zimbabwe and Zambia [12]. This has seen most being attended to by TBAs as they cannot afford hospital bills. More so, for women with physical disabilities, most healthcare infrastructure is physically inaccessible. Fortunately, sociocultural values as well as tradition in the two countries imbue TBAs power and authority to manage pregnancies and assist in child deliveries time immemorial [22-24].

Whilst this is so, Kruske, et al. [17] 's study in Zimbabwe 
noted that, the government policies expounded through the Ministry of Health programmes and policies appear to be relegating these TBAs to the fringes of healthcare provision. The argument is that, in a country with a failing health system characterized by mass exodus of qualified personnel, availability of drugs, and understaffing of healthcare centres, among others, TBAs remain the lifeline for poor women and this includes most women with disabilities. Thus the PWwDCCM can do justice by incorporating TBAs. Inclusion of TBAs in this model can be argued to be a viable solution towards achieving SDGs number 3 as well as promoting article number 25 of the United Nations Convention on the Right of Persons with Disabilities... In other words, in countries reeling with high maternal deaths as is the case with Zambia and Zimbabwe, TBAs' status and position in society make them the best intervention tools to be included in PWwDCCM [25].

The government and the Ministry of Health for both countries in question should capitalize on the availability of and standing working relations of TBAs with the grassroots for better/positive maternal health outcomes among women with disabilities. TBAs live with women with disabilities, they speak the local language, have the trust of community members, provide psychosocial support at birth, and are thus an integral part of African medicine. Hence leaving them out of the healthcare model can be argued to be a misnomer.

All that is called for is a multidisciplinary approach where TBAs in each community should be attached to a specialist or a coordinator for referral purposes if complications are suspected, for example, they can be attached to a coordinator based at a local clinic or district hospital. The coordinator can be assigned to certain area or ward thus will be coordinating those TBAs in her/his jurisdiction for referrals, workshops etc. This is to say, the model in question should be a decentralisation model which incorporates Traditional Birth Assistants. These people should be taken on board by Ministry of health and be trained on issues of hygiene and referrals.

Thus while both Zimbabwean and Zambian governments are overburdened financially to develop healthcare structures, they can adapt and modify existing healthcare structures in communities as is the case with clinics so that they become inclusive pregnant women with physical disabilities too. This would include rehabilitation of physical structures as well as enabling sanitary facilities such as water to be accessible for a person in wheelchair, toilets to have ramps and wider space to manure just to mention a few. Thus they may become accessible for referral purposes and this should apply to local hospitals. The model can be effective if TBAs are equipped with basic healthcare equipment by the Ministries concerned. These should include basic delivery equipment's and cell phones plus a small allowance to enable them to buy airtime or talk time. Decentralised and inclusive healthcare facilities to villages or communities where the needs of people are met immediately, is key in combating maternal deaths.

The village health workers model which the Ministry of Health in Zimbabwe put in place in the early 80s till now can be adopted and modified to suit the model for pregnant women with disabilities in both rural and cities. These village health workers mainly emphasise on community awareness pertaining to hygiene standards as well as attending to minor ailments among community members such as small cuts etc. Thus unlike the Village Health worker model, the PWwDCCM is supposed to be very sensitive to persons with disabilities especially pregnant women with disabilities. In other words, whilst the model would be inclusive of all women who cannot access sophisticated healthcare facilities due to financial constraints, physical barriers and distance, it should be more tailor made to pregnant women with disabilities [17]. Such model is likely to address challenges faced by most women with disabilities and their babies in accessing healthcare facilities as well as reducing maternal mortality which has been going unrecorded in the national health statistics due to unrecorded childbirths in Zambia, Zimbabwe and other African countries with similar challenges.

\section{References}

1. Chigunwe G (2014) Sanitary Provisions for Persons with Disabilities in the $21^{\text {st }}$ Century and Bridging the Gap through Collaborative Research. GJSS 4(2): 51-61.

2. WHO (2017) End preventable deaths: Global action plan for prevention and control of pneumonia and diarrhoea (GAPPD). Geneva: World Health Organization.

3. WHO (2015) Essential environmental health standards in health care. Geneva: World Health Organization.

4. World Bank, WHO (2011) World Report on Disability, Washington, DC.

5. Rugoho T, Maphosa F (2017) Challenges faced by women with disabilities in accessing sexual and reproductive health in Zimbabwe. The case of Chitungwiza town 6 african journal of disability 6: 252 .

6. Ganle JK, Otupiri E, Obeng B, Edusie AK, Ankomah A, Adamu R (2016) Challenges Women with Disabilities face in Accessing and Using Maternal Healthcare Services in Ghana: A Qualitative Study. PLos ONE 11(6): 20158361.

7. Chigunwe (2019) Big Doors Swing on Little Hinges: 
Access to Sanitary Facilities and Maternal Health. Journal of Epidemiology and Public Health Reviews 4(2): 8211174.

8. WHO (2018) Meeting the fundamental need for water, sanitation and hygiene services in health care facilities. Global meeting held in Madrid.

9. WHO (2014) Making Pregnancy Safer: The Critical Role of the Skilled Attendant: A Joint Statement by WHO, ICM and FIGO, Geneva: WHO.

10. WHO (2018) Reducing Inequalities in Health-proposals for health promotion policy and action, Consensus Statement, European Committee for Health Promotion Development, WHO Regional Office for Europe. Copenhagen.

11. Zambia Central Statistical Office (CSO) (2010) Ministry of Health, Tropical Disease Research Centre (TDRC), University of Zambia, and Macro International Inc. (2009) Zambia Demographic Health Survey 2007 Calverton, Maryland, USA: CSO and Macro International Inc.

12. UNDP (2011) Millennium Developmental Goals progress report 2011. Lusaka.

13. Morrison J, Basnet M, Bhudhathoki B, Adhikari D, Tumbahangphe $\mathrm{K}$, et al. (2014) Disabled Women's Maternal and New born Healthcare in Rural Nepal: A Qualitative Study. J Midw Nov 30(11): 1132-1139.

14. Kolinsky M, Timyan J, Gay J (2017) The Health of Women: Global Perspective Washington Westview Press.

15. Alwan K(2014) Raising the priority of non-communicable diseases in development work at global and national levels. Central Statistical Office Lusaka.

16. Gill C, Phiri Mazala G, Guerina NG, Kasimba J, Mulenga C, et al. (2011) Effect of training traditional birth attendants on neonatal mortality (Lufwanyama Neonatal Survival Project): randomised controlled study. BMJ 342: 346.

17. Kruske S, Barclay L (2014) Effect of shifting policies on traditionalbirth attendant training. Journal of Midwifery and Women's Health 49(4): 306-311.

18. Firoz T, Vidler M, Makanga PT, Boene H, Chiau R, et al. (2016) Community Perspectives on the Determinants of Maternal Health in the Rural Southern Mozambique: A Qualitative Study.Reproductive Health 13(S2): 112.

19. Graham S (2000) Traditional Birth Attendants in Karamoja, Kampala: South Bank University.

20. United Nations Development Programme (UNDP) (2011) Zambia Human Development Report 2011. Service delivery for sustainable human development. United Nation Development Programme Lusaka.

21. Zimbabwe The Ministry of Health's National Health Strategy document for 2016-2020.

22. http://doi.org/10.1371/journal (2020).

23. (2020) Women-seeking-abortion.

24. UN (2010) General Assembly Resolution The human right to water and sanitation.

25. (2007) Zimbabwe Central Statistical Office and Macro International, Zimbabwe Demographic and Health Survey Central Statistical Office and Macro International, New York, USA. 